\title{
Antropología
}

\section{La asistencia personal como nuevo derecho de ciudadanía: una mirada desde el ámbito de los profesionales de la salud}

Personal assistance as a new citizens' right: seen from the health professionals' sphere

Assistência pessoal como novo direito de cidadania: um olhar do campo dos profissionais de saúde

\author{
Rubén González-Rodríguez ${ }^{1}$ Carmen Verde-Diego², Violeta Pérez-
}

Lahoz $^{3}$

\begin{abstract}
${ }^{1}$ Doctor por la Universidad de Santiago de Compostela. Universidad de Vigo, Ourense, España. Correo electrónico: rubgonzalez@uvigo.es

${ }^{2}$ Doctora por la Universidad de Santiago de Compostela. Universidad de Vigo, Ourense, España. Correo electrónico: carmenverde@uvigo.es

${ }^{3}$ Doctoranda. Universidad de Vigo, Ourense, España. Correo electrónico: violeta@uvigo.es)

Cómo citar este artículo en edición digital: González-Rodríguez, R., Verde-Diego, C. E Pérez-Lahoz, V.

(2019). La asistencia personal como nuevo derecho de ciudadanía: una mirada desde el ámbito de los profesionales de la salud. Cultura de los Cuidados (Edición digital), 23(53). Recuperado de http://dx.doi.org/10.14198/cuid.2019.53.11

Correspondencia: Rubén González-Rodríguez. Facultade de Ciencias da Educación. Campus das Lagoas, s/n. Universidad de Vigo. 32004 Ourense-España

Correo electrónico: rubgonzalez@uvigo.es

Recibido: 26/11/2018; Aceptado: 10/01/2019
\end{abstract}

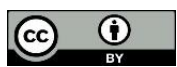

\section{ABSTRACT}

Objective: The main objective of this research is to familiarize ourselves with the figure of the personal assistant for disabled people. It also seeks to reveal the importance of professionalized care and accompaniment compared with family care.

Methods: Joint research consisting of the review of official databases, as well as the performance of semi-structured interviews with 11 professional nurses belonging to the
Public Health Service of Galicia (Spain).

Results: The results suggest that the figure of the personal assistant is still not predominant in the provision of services for people with disabilities. The interviewed nursing staff considered that the functions of personal assistance should be clearly professionalized due to their specific characteristics.

Conclusions: The results of this study constitute a framework for reflection on 
intervention and support for people with disabilities. No doubt, interdisciplinary work is necessary within a joint approach of professionals with health and social training

Keywords: People with disabilities; family; nursing; activities of daily living.

\section{RESUMO}

Objetivo: $\mathrm{O}$ objetivo principal desta pesquisa é conhecer a figura do assistente pessoal para pessoas com deficiência. Da mesma forma, pretende-se expor a importância do cuidado e o acompanhamento profissionalizado versus cuidado familiar.

Método: Abordagem de pesquisa mista composta pela revisão de bases de dados oficiais, bem como entrevistas semiestruturadas a 11 profissionais de enfermagem pertencentes ao Serviço de Saúde Pública da Galiza (Espanha).

Resultados: Os resultados sugerem que a figura do assistente pessoal continua sem ser uma maioria na prestação de serviços para pessoas com deficiência. A equipe de enfermagem entrevistada considera que as funções de assistência pessoal devem estar focadas em uma profissionalização clara devido às suas características específicas.

Conclusões: Os resultados deste estudo constituem um marco adequado para a reflexão sobre a intervenção e acompanhamento de pessoas com deficiência. Sem dúvida, o trabalho interdisciplinar é necessário dentro de uma abordagem conjunta de profissionais com formação em saúde e social

Palavras chave: Pessoas com deficiência; família; enfermagem; atividades cotidianas.

\section{RESUMEN}

Objetivo: El objetivo principal de esta investigación es conocer la figura del asistente personal para personas con discapacidad. Asimismo, se pretende exponer la importancia de los cuidados y el acompañamiento profesionalizado frente a los cuidados familiares.

Método: Abordaje de investigación mixto consistente en la revisión de bases de datos oficiales, así como la realización de entrevistas semiestructuradas a 11 profesionales de la enfermería pertenecientes al Servicio Público de Salud de Galicia (España).

Resultados: Los resultados apuntan a que la figura del asistente personal continúa sin ser mayoritaria en la prestación de servicios para personas con discapacidad. El personal de enfermería entrevistado considera que las funciones de asistencia personal deben enfocarse hacia una clara profesionalización por sus características específicas.

Conclusiones: Los resultados de este estudio, constituyen un marco propio para la reflexión sobre la intervención $y$ acompañamiento en personas con discapacidad. Sin duda, es necesario el trabajo interdisciplinar dentro de un abordaje conjunto de profesionales con formación sanitaria y social

Keywords: Personas con discapacidad; familia; enfermería; actividades de la vida diaria.

\section{INTRODUCCIÓN}

Los últimos datos sobre el número de personas con discapacidad en España, según la encuesta Discapacidad, Autonomía Personal y Dependencia, estiman una población alrededor de 3,84 millones en el año 2007 (Instituto Nacional de Estadística [INE], 2008). Si bien la edad no implica necesariamente una situación de discapacidad o dependencia, si es verdad que con el aumento de la edad se observa 
una mayor limitación de la capacidad funcional (Pereira, 2003). Así, de los 3,84 millones a los que previamente se hacía referencia, 2,2 millones son personas de más de 65 años y alrededor de la mitad, personas de 80 y más años (INE, 2008). Como vemos, una población significativa que necesita en muchos casos prestaciones e intervenciones desde diferentes sistemas de protección social, especialmente desde el Sistema de salud y el Sistema de servicios sociales.

En España, no se crea un sistema de protección específico para atender las situaciones de dependencia hasta el año 2007, mediante la Ley 39/2006, de 14 de diciembre, de promoción de la autonomía personal y atención a personas en situación de dependencia. Previamente, la protección a las personas mayores y/o con discapacidad, que precisaban de la asistencia de otra persona, se hacía desde otros sistemas de protección social ya existentes (Sempere, 2008).

Si bien las situaciones de discapacidad y dependencia no son equivalentes (González-Rodríguez, 2017), bien es verdad que la dependencia supone el apoyo de una tercera persona para el desarrollo de las actividades básicas de la vida diaria (ABVD) (Sánchez-Urán, 2009). Por ello, las personas con diversidad funcional constituyen una parte importante de los beneficiarios del Sistema para la Autonomía y Atención a la Dependencia en España (SAAD).

Dentro de su catálogo de servicios encontramos diferentes tipos de prestaciones. Si las vinculamos con las diferentes tipologías del cuidado que postula Carrasco (2006) podemos establecer las siguientes vías mediante las que se concreta la atención: mercado, sector público y trabajo no remunerado. Según Carrasco (2006), la vía principal de atención en España, es decir, la más representativa, se materializa a través del trabajo no remunerado que prestan a la persona en situación de dependencia personas de su red familiar e informal. De forma más pormenorizada Fernández-de-Larrinoa et al. (2011) detallan que el perfil más frecuente de persona cuidadora corresponde a una mujer de 60 años aproximadamente que, a causa de llevar años realizando esta labor, muestra una percepción de su salud peor que el resto de la población, derivando en una peor calidad de vida.

Dentro del catálogo del SAAD podemos identificar 3 prestaciones que implican unas intervenciones de apoyo en la realización de las actividades de la vida diaria para personas beneficiarias del sistema y que residen en sus hogares: 1) el servicio de ayuda a domicilio; 2) la prestación económica de asistencia personal; y 3) la prestación económica de cuidados familiares.

El servicio de ayuda a domicilio es claramente un servicio profesionalizado, prestado por entidades públicas o bien por entidades privadas, que realizan apoyo en actividades de carácter personal y/o doméstico en el propio domicilio de la persona en situación de dependencia. Los/las profesionales que desempeñan esta ocupación laboral cuentan con titulación de formación profesional o bien con un certificado de profesionalidad equivalente.

La prestación económica de cuidados familiares supone una cuantía monetaria dirigida a "soportar" los gastos directos extraordinarios que la persona en situación de dependencia tiene a diario. El beneficiario de la prestación es la persona dependiente y no la persona cuidadora. Por ello, cumple los requisitos de cuidado informal que postulan Andersson, Levin y Emtinger (2002): (a) es desarrollado por personas de la red social del receptor del 
cuidado y (b) se provee de forma voluntaria, sin que medie ninguna organización ni remuneración. En este último caso, solo tendrá cabida una remuneración o compensación de tipo claramente ocasional (Rogero, 2010). En esta tipología de cuidados informales continúa prevaleciendo con mayor representatividad el género femenino. Así, según los datos facilitados por el IMSERSO, referidos a 31 de marzo de 2018, el perfil de las personas cuidadoras con reconocimiento de esta prestación está configurado en un $88,8 \%$ por mujeres y un $11,2 \%$ de hombres 1 . Mayobre y Vázquez (2015) esgrimen diferentes causas tales como prescripciones de género, posición de clase, obligaciones sociales o imperativos morales, factores que se interiorizan en el proceso de socialización y que les impiden a las mujeres, en muchas ocasiones, plantearse la posibilidad de elegir.

El tercer foco de análisis es la prestación de asistencia personal (PEAP). La asistencia personal es un servicio de apoyo a las personas con diversidad funcional, no desarrollado en el ámbito de los servicios sociales de España hasta hace poco, pero que lleva más de 30 años establecido en países como EE.UU., Suecia, Gran Bretaña, Noruega, Brasil, etc. (Rueda, 2013).

En el mundo de la diversidad funcional, una de las organizaciones que más se ha movilizado por la puesta en práctica de la figura de asistencia personal fue, y continúa siendo en la actualidad, el Movimiento de Vida Independiente. Este movimiento, surgido en Estados Unidos (Berkeley) en los años 60, entiende la asistencia personal como un apoyo para las personas con diversidad funcional, para así facilitar la recuperación de su autonomía (Carbonell, 2017). Rodríguez-Picavea define al asistente personal como "aquella persona que ayuda a otra a desarrollar su vida, esto es, que realiza o ayuda a realizar las tareas de la vida diaria a otra persona que, por su situación, bien sea por una diversidad funcional, o por otros motivos, no puede realizarlas por sí misma" (2007, p. 117). Se trata, por tanto, de una figura de apoyo (Arnau, 2006; Planas, 2013) que, asimismo, tiene como objetivo el empoderamiento de las personas con diversidad funcional o en situación de dependencia (Suñe y MartínezGarcía, 2015).

$\mathrm{Al}$ respecto de las tareas que desarrolla un asistente personal, siguiendo a RodríguezPicavea y Romanach (2006) podemos dividirlas y agruparlas en diferentes áreas: 1) tareas personales; 2 ) tareas del hogar; 3 ) tareas de acompañamiento; 4) tareas de conducción; 5) tareas de comunicación; 6) tareas de coordinación; 7) tareas excepcionales; y 8) tareas especiales.

Oliveira, Barbosa, Fernandes y Fernandes (2017) indican que debe mejorarse la promoción de la autonomía, tanto en las personas con diversidad funcional como con las personas mayores, así como una mejor comprensión de sus necesidades individuales, principio que genera un consenso mayoritario entre la comunidad científica. La prestación de asistencia personal supone, en línea con este posicionamiento, un apoyo más amplio que en las propias tareas domésticas o en los cuidados personales, como son por ejemplo la planificación del día a día y la toma de decisiones, apoyo en situaciones especiales de crisis, acompañamientos específicos, así como el apoyo en actividades propias del área afectivo-sexual (Gónzalez-Rodríguez, Gandoy y Verde, 2014).

La finalidad principal de este trabajo es conocer la implantación en España de la figura del asistente personal para personas con diversidad funcional. Pretendemos, asimismo, analizar si es una figura conocida entre los profesionales de la enfermería, así 
como explorar si lo consideran un servicio profesionalizado (frente a los cuidados familiares) que pueda suponer un nuevo nicho de trabajo para este colectivo profesional.

\section{METODOLOGÍA}

Para la realización de nuestra investigación se utilizó un abordaje mixto. Por una parte, una explotación de datos secundarios de organismos oficiales para su posterior análisis. Asimismo, se realizó un estudio cualitativo donde se recogieron datos a través de entrevistas semiestructuradas a 11 profesionales de la enfermería. Este personal de enfermería (10 mujeres y 1 hombre, con edades comprendidas entre los 25 y los 53 años) trabaja en el Sistema de salud público de la región de Galicia (noroeste de España). Todos ellos fueron invitados a participar de forma telefónica $\mathrm{y}$, previamente a ser entrevistados, prestaron un consentimiento informado por escrito donde se les explicaban los objetivos y alcance de la investigación a realizar.

\section{RESULTADOS \\ Características de la muestra}

Las principales características sociodemográficas y profesionales de la población objeto de estudio se presentan en la tabla 1. Como puede observarse, la muestra final resultante la conforman 11 enfermeros/as, de las cuales 10 eran mujeres (90,9\%). Sus edades estaban comprendidas entre los 25 y los 53 años (M=40; DT=9,7).

Respecto al nivel máximo de estudios alcanzado, prácticamente la mitad (45,45\%) están en posesión únicamente de la diplomatura universitaria en enfermería. Dos han obtenido el título de grado en enfermería y cuatro han alcanzado el segundo ciclo universitario (llegando una de ellas a la obtención del doctorado a través de un programa de Antropología Social y Cultural). Como se ha hecho referencia en el apartado Metodología, todos los sujetos que componen la muestra son trabajadores/as en el Servicio público de salud de Galicia. El $45,45 \%$ trabajan en atención primaria de salud y el 54,55\% en servicios o unidades correspondientes a atención especializada (tanto hospitalaria como ambulatoria).

TABLA 1: Características sociodemográficas y laborales de la muestra

\begin{tabular}{clccl}
\hline $\begin{array}{c}\text { N. }{ }^{\text {de }} \\
\text { entrevista }\end{array}$ & Género & Edad & $\begin{array}{c}\text { Mayor grado académico } \\
\text { alcanzado }\end{array}$ & $\begin{array}{c}\text { Nivel de atención en el } \\
\text { que trabaja }\end{array}$ \\
\hline E1 & Mujer & 25 & Grado & Atención primaria \\
E2 & Mujer & 53 & Diplomatura & Atención primaria \\
E3 & Mujer & 46 & Diplomatura & Atención primaria \\
E4 & Mujer & 34 & Máster & Atención especializada \\
E5 & Mujer & 27 & Grado & Atención especializada \\
E6 & Mujer & 49 & Diplomatura & Atención especializada \\
E7 & Hombre & 36 & Máster & Atención primaria \\
E8 & Mujer & 43 & Doctorado & Atención especializada \\
E9 & Mujer & 47 & Diplomatura & Atención especializada \\
E10 & Mujer & 51 & Diplomatura & Atención especializada \\
E11 & Mujer & 29 & Máster & Atención primaria \\
\hline
\end{tabular}




\section{Implantación de la figura de asistente personal en España}

Como se exponía con anterioridad, el servicio de asistencia personal es una prestación reconocida recientemente desde el ámbito público a través del SAAD y, por tanto, con poco recorrido en la cotidianidad de los profesionales que realizan intervenciones en el ámbito sociosanitario.

$\mathrm{Si}$ bien inicialmente se apuntaba a su desconocimiento por parte de los profesionales, se esperaba un mayor desarrollo en su implantación. Sin embargo, si observamos la evolución en las prestaciones resueltas en el año 2013, respecto a la actualidad del 2018, apenas observamos diferencias significativas. Prestaciones como la Teleasistencia o la Ayuda a domicilio se incrementaron en 3 y 4 puntos respectivamente. Por el contrario, el servicio de asistencia personal pasó de un ínfimo $0,15 \%$ a un $0,54 \%$. Continúa siendo la última prestación reconocida de entre todas las incluidas en el catálogo de prestaciones y servicios del SAAD (tabla 2).

\section{TABLA 2: Prestaciones reconocidas dentro del Sistema para la Autonomía y Atención a la Dependencia}

\begin{tabular}{lrrrr}
\hline \multicolumn{1}{c}{ Tipología de prestación } & \multicolumn{2}{c}{} & \multicolumn{2}{c}{$\mathbf{2 0 1 8}$} \\
& N. & \multicolumn{1}{c}{$\boldsymbol{\%}$} & \multicolumn{1}{c}{ N. } & \multicolumn{1}{c}{$\%$} \\
\hline Prevención dependencia y promoción de la autonomía personal & 20.994 & 2,22 & 48.453 & 4,05 \\
Teleasistencia & 120.457 & 12,75 & 188.518 & 15,75 \\
Ayuda a domicilio & 120.962 & 12,80 & 202.407 & 16,91 \\
Centros de día/noche & 70.343 & 7,44 & 91.460 & 7,64 \\
Atención residencial & 130.424 & 13,80 & 158.457 & 13,24 \\
Prestación económica vinculada al servicio & 72.029 & 7,62 & 114.716 & 9,58 \\
Prestación económica de cuidados familiares & 408.401 & 43,21 & 386.511 & 32,29 \\
Prestación económica de asistencia personal & 1.441 & 0,15 & 6.463 & 0,54 \\
\hline
\end{tabular}

Nota.- FUENTE: Datos estadísticos SAAD (IMSERSO, diciembre 2013; marzo 2018)²

Asimismo, si observamos el ámbito territorial donde se están resolviendo estos servicios observamos que, con una diferencia notable, es el País Vasco la comunidad autónoma donde las resoluciones son más significativas, representando un $85,97 \%$ respecto de la totalidad de prestaciones de asistencia personal reconocidas en el conjunto del Estado. Si a País Vasco sumamos Castilla y León $(10,34 \%)$, prácticamente representan todas las prestaciones reconocidas (tabla 3).
Conocimiento del servicio de asistencia personal por parte del personal de enfermería

Las respuestas dadas por el personal de enfermería entrevistado, respecto al conocimiento del servicio de asistencia personal, fueron muy poco homogéneas en muchas ocasiones. Así, mayoritariamente identificaban el servicio de asistencia personal con el servicio de ayuda a domicilio. Una de las entrevistadas manifestaba que " $n o$ era conocedora de las diferentes tipologías de servicios y entendía que representaban la misma prestación, siendo proporcionado siempre por personal de ayuda a domicilio" (E9). 
TABLA 3: Prestaciones reconocidas de asistencia personal en el SAAD, según el ámbito territorial

\begin{tabular}{lrc}
\hline \multicolumn{1}{c}{ Ámbito Territorial } & N & $\mathbf{2 0 1 8} \mathbf{2}$ \\
\hline Andalucía & 9 & 0,00 \\
Aragón & 0 & 0,00 \\
Asturias (Principado de) & 1 & 0,00 \\
Illes Balears & 0 & 0,00 \\
Canarias & 0 & 0,00 \\
Cantabria & 0 & 0,00 \\
Castilla y León & 668 & 0,57 \\
Castilla-La Mancha & 19 & 0,03 \\
Catalunya & 19 & 0,01 \\
Comunitat Valenciana & 6 & 0,01 \\
Extremadura & 0 & 0,00 \\
Galicia & 104 & 0,16 \\
Madrid (Comunidad de) & 79 & 0,05 \\
Murcia ( Región de) & 0 & 0,00 \\
Navarra (Comunidad Foral de) & 2 & 0,01 \\
País Vasco & 5.556 & 7,38 \\
La Rioja & 0 & 0,00 \\
Ceuta y Melilla & 0 & 0,00 \\
TOTAL & 6.463 & 0,54 \\
\hline
\end{tabular}

Nota.- FUENTE: Datos estadísticos SAAD (IMSERSO, marzo 2018)²

La totalidad de las personas entrevistadas asumían que las tareas a realizar, propias de un asistente personal, suponían el apoyo en la realización de las actividades básicas de la vida diaria tales como aseo, alimentación y vestido. Sin embargo, solo tres de ellos (E1, E4 y E8) hacían referencia al respecto del apoyo en la autonomía en la toma de decisiones, acompañamientos en los ámbitos educativo y/o laboral y apoyo en la esfera afectivo-sexual. Asimismo, todos ellos diferenciaban claramente que el servicio de asistencia personal no tiene nada que ver con otros servicios de apoyo a nivel domiciliario y con intervenciones en mayor medida competencia de los servicios de salud, tales como la prestación de enfermería de atención primaria de salud en el domicilio de la persona $u$ otras prestaciones de salud como la hospitalización domiciliaria.

$\mathrm{Al}$ respecto de la edad de las personas en situación de dependencia, resultó significativo que ninguno de ellos contempló la posibilidad de que la persona que necesitaba apoyo pudiese ser una persona menor de edad, esto es, encuadrada en el colectivo etario de infancia y adolescencia. Si bien algunos de ellos/as contemplaron únicamente la prestación para personas jóvenes con discapacidad (E1, E4, E7 y E11), el resto de entrevistados entendió que los beneficiarios de la prestación podrían ser personas con 
diversidad funcional, así como personas mayores con necesidades de apoyo específicas.

Una vez explicada la finalidad, objeto y beneficiarios de la prestación de asistencia personal, se indagó sobre qué perfil de titulados/as debería prestar ese servicio. En este punto, sí entendían mayoritariamente que debía exigirse una titulación superior del ámbito social y/o sanitario, como por ejemplo enfermería. Esgrimían argumentos tales como: "es necesaria una formación específica para intervenir en determinados procesos que puedan suponer una urgencia, por ejemplo, un paciente con medidas de apoyo tales como alimentación artificial u oxigenoterapia" (E10). Además, en otros perfiles de beneficiario como el caso de personas con enfermedad mental, otra enfermera manifestaba que "fíjate si es específico que, la atención en enfermedad mental es una especialización propia dentro de enfermería (EIR). Entiendo que quien intervenga con situaciones tan específicas debe tener no solo un conocimiento previo del abordaje clínico adecuado sino también de los procesos de intervención e integración comunitaria" (E11). Dos de las entrevistadas (E4 y E8) resaltaban la tarea de apoyo en la toma de decisiones. Manifestaban que estas funciones tan específicas podrían suponer dilemas éticos importantes, por lo que entendían que "para suscitar este tipo de dudas en las intervenciones, era necesario que la formación previa de los profesionales incluyese cuestiones de ética y deontología profesional".

Todos ellos manifestaron que consideraban que, entre todas las titulaciones universitarias del ámbito social y sanitario, entendían que también el personal de enfermería podría ser susceptible de desempeñar las funciones de un asistente personal, si bien situaban un encaje vinculado más a patologías físicas y que precisasen, en mayor grado, medidas de apoyo en salud específicas. En ningún caso se aceptó considerar este servicio dentro del marco de los cuidados informales, entendiendo además que debería ser prestado bien por la administración pública bien por entidades acreditadas especializadas.

\section{DISCUSIÓN Y CONLUSIONES}

Como podemos comprobar, las vías familiares de cuidado continúan representando un porcentaje elevado de entre los cuidados que se prestan a las personas en situación de dependencia (entre el $30-40 \%)^{1}$. Por lo tanto, podemos afirmar que los cuidados informales no han sido desplazados por la cobertura de servicios prestados por las Administraciones públicas o por las entidades de naturaleza privada.

De entre los nuevos servicios que ofrecen prestaciones diferentes a las ya clásicamente reconocidas dentro del ámbito sociosanitario se encuentra la de asistencia personal. Sin embargo, parece que solo País Vasco (y Castilla y León en menor medida) han realizado una apuesta clara y específica por el reconocimiento de esta prestación. López-Pérez (2012) realizó un estudio sobre la implantación de la PEAP y ya constataba que el desarrollo de esta prestación resultaba insuficiente. Apuntaba principalmente a la falta de desarrollo normativo específico, la falta de presupuesto económico y también a la falta de voluntad política.

González-Rodríguez et al. (2014) indican que, en el caso del País Vasco, esta voluntad política posibilitó el empuje hacia un reconocimiento de esta prestación para potenciar los derechos y la autonomía de las personas con diversidad funcional. Así, en un estudio realizado en la provincia de 
Guipúzcoa se ha determinado que, de las 923 solicitudes de PEAP registradas hasta noviembre de 2013, 310 eran "migraciones" de prestaciones económicas de cuidados familiares a prestaciones de asistencia personal (Benedicto, 2013).

A pesar de que, en las últimas décadas, el modelo social y de reconocimiento de derechos para las personas con diversidad funcional va ganando espacio al tradicional modelo médico-rehabilitador entre el profesional técnico y las diferentes instituciones, parece que la materialización de este cambio continúa siendo demasiado lento.

Dadas sus características complejas y que exigen una formación específica importante, es recomendable que los profesionales que realizan funciones de asistencia personal estén debidamente capacitados. En línea con lo mentado por Matus-López (2015), el no control sobre la cualificación del profesional puede suponer una asistencia de baja calidad, llegando incluso a tener consecuencias para ambas partes. Los titulados en enfermería, junto con otros profesionales del ámbito social y/o sanitario, poseen la capacitación necesaria para ejercer este tipo de tareas.

Parece necesario realizar un esfuerzo para difundir e informar sobre la figura del asistente personal, tanto a las familias como entre el profesional técnico que realiza intervenciones en el marco sociosanitario. Sin duda, facilitar la accesibilidad a este tipo de prestaciones supone garantizar $y$ extender el reconocimiento de derechos para las personas con diversidad funcional, para garantizar la igualdad y la integración en la sociedad en condiciones equiparables al resto de la ciudadanía.

\section{BIBLIOGRAFÍA}

- Andersson, A., Levin, L-A., y Emtinger, B.G. (2002). The economic burden of informal care. International Journal of Technology Assessment in Health Care, 18(1), 46-54.

- Arnau, M. S. (2006). Ley de la "in-Dependencia": pasos necesarios para garantizar un nuevo derecho de ciudadanía. Lan Harremanak, 15(2), 41-64. Recuperado de http://www.ehu.eus/ojs/index.php/ Lan_Harremanak/article/view/3116.

- Benedicto, Z. (2013). La prestación económica de asistencia personal en el territorio histórico de Gipuzkoa. Zerbitzuan, 54, 67-84. Recuperado de http://dx.doi.org/10.5569/1134-7147.54.04.

- Carbonell, G. J. (2017). El asistente personal para una vida independiente: una figura en construcción. Revista de Derecho UNED, 21, 187-216. Recuperado de http://revistas.uned.es/index.php/RDUNED/article/v iew/21180/17477.

- Carrasco, C. (2006). Presentación. Revista de Economía Crítica, 5, 5-6.

- Fernández-de-Larrinoa, P., Martínez-Rodríguez, S., Ortiz-Marqués, N., Carrasco, M., Solabarrieta, J., y Gómez-Marroquín, I. (2011). Autopercepción del estado de salud en familiares cuidadores y su relación con el nivel de sobrecarga. Psicothema, 23(3), 388-393.

- González-Rodríguez, R. (2017). Discapacidad vs Dependencia. Terminología diferencial y procedimiento para su reconocimiento. Índex de Enfermería, 26(3), 170-174.

- González-Rodríguez, R., Gandoy, M. y Verde, C. (2014). Aproximación al servicio de asistencia personal y su implantación en España. Perfil del beneficiario de la prestación en Galicia. Alternativas: Cuadernos de Trabajo Social, 21, 119-141.

- Instituto Nacional de Estadística. (2008). Encuesta sobre discapacidades, autonomía personal y situaciones de dependencia. Disponible en http://www.ine.es/dyngs/INEbase/es/operacion.htm ?c=Estadistica_C\&cid=1254736176782\&menu=resulta dos\&idp $=1254735573175$.

- Ley 39/2006, de 14 de diciembre, de promoción de la autonomía personal y atención a las personas en situación de dependencia. Boletín Oficial del Estado, 299, de 15 de diciembre de 2006.

- López-Pérez, M. (2012). Prestación económica de asistencia personal: asignatura pendiente de la Ley 39/2006, de 14 de diciembre. Portularia, 12(Extra), 111120. 


\section{Cultura de los Cuidados}

- Matus-López, M. (2015). Pensando en políticas de cuidados de larga duración para América Latina. Salud Colectiva, 11(4), 485-496. Recuperado de http://dx.doi.org/10.18294/sc.2015.785.

- Mayobre, P. y Vázquez, I. (2015). Cuidar cuesta: un análisis del cuidado desde la perspectiva de género. Revista Española de Investigaciones Sociológicas, 151, 83-100. Recuperado de http:// dx.doi.org/10.5477/cis/reis.151.83.

- Oliveira, S. M., Barbosa, K. T. F., Oliveira, M. R. L., Fernandes, W. A. A. B., y Fernandes, M. G. M. (2017). Determinantes sociales, económicos y ambientales de envejecimiento activo en ancianos atendidos en un centro de atención integral a la salud. Cultura de los Cuidados (Edición Digital), 21(49). Recuperado de http://dx.doi.org/10.14198/cuid.2017.49.17.

- Pereira, C. (2003). Envelhecimento com dependencia: responsabilidades e demandas da familia. Cadernos de Saúde Pública, 19(3), 733-781. Recuperado de http://dx.doi.org/10.1590/S0102-311X2003000300009.

- Planas, M. J. (2013). El asistente personal para las personas con diversidad funcional: una herramienta para el cumplimiento de derechos. Trabajo Social Hoy, 1(68), 31-54. Recuperado de https:// dialnet.unirioja.es/servlet/articulo? codigo $=52 \underline{54333}$.

- Rodríguez-Picavea, A. (2007). Reflexiones en torno a la figura del asistente personal para la vida independiente y la promoción de la autonomía de las personas con diversidad funcional (discapacidad). Zerbitzuan, 41, 115-128. Recuperado de https://dialnet.unirioja.es/descarga/articulo/2335334. pdf.

- Rodríguez-Picavea, A., y Romanach, J. (2006). Consideraciones sobre la figura del Asistente Personal en el Proyecto de la Ley de Promoción de la Autonomía Personal y Atención a las Personas en Situación de Dependencia.

Recuperado de http:// www.carm.es/ctra/cendoc/haddock/14123.pdf.
- Rogero, J. (2010). Los tiempos del cuidado. El impacto de la dependencia de los mayores en la vida cotidiana de sus cuidadores. Madrid: Instituto de Mayores y Servicios Sociales.

- Rueda, M. (2013). Análisis comparativo de las iniciativas de vida independiente en España. Recuperado de http://www.forovidaindependiente.org.

- Sánchez-Urán, Y. (2009). La nueva protección de la dependencia: naturaleza. En R. Roqueta (Coord.). La situación de dependencia: Régimen jurídico aplicable tras el desarrollo estatal y autonómico de la Ley de Dependencia (pp. 29-57). Valencia: Tirant lo Blanch.

- Sempere, A. V. (2008). Presupuestos, antecedentes y gestación de la Ley. En A.V. Sempere (Dir.). Comentario sistemático a la Ley de la Dependencia (pp. 77112). Navarra: Thomson-Aranzadi.

- Suñe, A. y Martínez-García, I. (2015). La figura del asistente social. En E. Ortega (Coord.). Situación de la Asistencia Personal en España (pp. 18-22). Madrid: Plataforma representativa estatal de personas con discapacidad física. Recuperado de http://www.predif.org/sites/default/files/documents/ ASISTENCIA\%20PERSONAL.pdf.

\section{NOTAS}

(1) Accesibles en http://www.dependencia.imserso.es/dependencia 01/s aad/sisaad/estadisticas sisaad/index.htm.

(2) El porcentaje indicado en cada uno de los ámbitos territoriales no corresponde al porcentaje relativo del total de prestaciones del Estado, sino al porcentaje relativo de resoluciones de asistencia personal en cada una de las comunidades autónomas. 